\title{
DESIGUALDADES SOCIOECONÔMICAS NA MORTALIDADE POR CÂNCER DE MAMA: REVISÃO SISTEMÁTICA
}

\author{
TORTAJADA, Juliana dos Santos ${ }^{1}$ \\ OLIVEIRA, Thaliany Siqueira ${ }^{1}$ \\ COSTA, Cássia Kely Favoretto ${ }^{2}$ \\ PICININ, Marcelo Bernuci ${ }^{3}$ \\ MASSUDA, Ely Mitie ${ }^{4}$
}

RESUMO: O câncer de mama é uma das neoplasias mais frequentes no mundo e a condição socioeconômica exerce influência na sobrevida das mulheres. Investigar as associações entre nível socioeconômico na incidência por câncer de mama. Revisão sistemática por meio de pesquisa de estudos indexados nas bases de dados Biblioteca Virtual em Saúde (BVS), Scielo, Medline, Biblioteca Cochrane, LILACS e US National Library of Medicine National Institutes of Health (PUBMED). Esta revisão foi desenvolvida conforme as recomendações metodológicas da declaração PRISMA - Preferred Reporting Items for Systematic Reviews and Meta-Analyses. O nível socioeconômico influencia significativamente as práticas de prevenção secundária do câncer de mama, de modo que quanto mais alto o nível socioeconômico da mulher, maior a prevalência de realização das condutas preventivas.

Palavras-chave: Nível socioeconômico. Neoplasias da mama. Classe social. Mortalidade.

\section{SOCIOECONOMIC INEQUALITIES IN BREAST CANCER MORTALITY: A SYSTEMATIC REVIEW}

SUMMARY: Breast cancer is one of the most frequent neoplasms in the world, and socioeconomic status influences the survival of women. To investigate associations between socioeconomic status in the incidence of breast cancer. Systematic review through research of indexed studies in the databases Virtual Health Library (VHL), Scielo, Medline, Cochrane Library, LILACS and US National Library of Medicine National Institutes of Health (PUBMED). This review was developed in accordance with the methodological recommendations of the PRISMA Preferred Reporting Items for Systematic Reviews and Meta-Analyzes. The socioeconomic level significantly influences the secondary prevention practices of breast cancer, so that higher the socioeconomic status of women, the higher the prevalence of preventive behaviors.

Keywords: Socioeconomic status. Breast neoplasms. Social class. Mortality.

\section{INTRODUÇÃO}

O câncer de mama é a segunda neoplasia mais frequente no mundo entre as mulheres, correspondendo a $25 \%$ de novos casos a cada ano (BRASIL, 2019). É um grave problema de saúde pública, pelo crescente número de casos diagnosticados e pelo investimento financeiro demandado

\footnotetext{
${ }^{1}$ Médica.

${ }^{2}$ Doutora em Economia Aplicada. Docente permanente do Programa de Pós-Graduação em Ciências Econômicas. Universidade Estadual de Maringá. Departamento de Economia

${ }^{3}$ Doutor em Fisiologia. Docente permanente do Programa de Pós-Graduação em Promoção da Saúde. Unicesumar -

Centro Universitário de Maringá. Programa de Pós-Graduação em Promoção da Saúde/ICETI

${ }^{4}$ Doutora em História Econômica. Docente permanente do Programa de Pós-Graduação em Promoção da Saúde;

Coordenadora do Programa de Pós-Graduação em Gestão do Conhecimento nas Organizações. Unicesumar - Centro

Universitário de Maringá/ICETI.
} 
para a detecção, prevenção e tratamento (FELIX et al., 2012).

A taxa de mortalidade por câncer de mama em países desenvolvidos vem apresentando tendência decrescente, embora haja um aumento na incidência desta doença. Este fato está ocorrendo em virtude do avanço nos meios de detecção precoce e melhorias nos tratamentos (CINTRA; GUERRA; BUSTAMANTE-TEIXEIRA, 2008). Entretanto, nos países em desenvolvimento, como o Brasil, a mortalidade e a incidência por câncer de mama continuam elevadas (GONÇALVES et al., 2007; FACINA, 2014).

A taxa de sobrevida da população feminina está diretamente associada à prevalência dos fatores de risco, a condição socioeconômica e aos fatores prognósticos (HÖFELMANN et al., 2014). Dentre os fatores de riscos existentes, os não modificáveis (idade, história familiar, gestação tardia, baixa paridade, entre outros) que são aqueles que mais influenciam no surgimento de câncer de mama. Como esses fatores são menos passíveis a intervenções de saúde pública, o rastreamento bem como o diagnóstico e tratamento precoce são indispensáveis para redução da mortalidade devido à doença (SILVA et al., 2014).

A população com baixo nível de escolaridade e pertencente ao nível socioeconômico baixo são mais comumente diagnosticados em estágios mais avançados da doença e, consequentemente, apresentam uma sobrevida menor, em comparação a classes sociais mais altas. Essa disparidade pode ser explicada pela diferença no acesso a serviços de boa qualidade, resolutividade e tempo prolongado até o diagnóstico e tratamento nestes países (OLIVEIRA et al., 2011; MATOS et al., 2010).

O diagnóstico na fase inicial, além de reduzir a mortalidade aumenta a chance de a doença não ter invadido linfonodos axilares, melhorando o prognóstico, possibilitando tratamentos menos agressivos e sequelas físicas e psicológicas (PEREIRA et al., 2014). Em mulheres que apresentam a neoplasia mamária, um determinante de doença avançada é a moradia em áreas no qual os serviços de saúde não existem ou não são eficientes (BARRY, 2005), impondo barreira importante à prevenção e diagnóstico precoce da doença RENCK et al., 2014).

Assim, torna-se necessária a compreensão dos fatores socioeconômicos na mortalidade por câncer de mama, buscando-se alcançar eficácia dos programas de prevenção secundária, principalmente o rastreio por meio da mamografia (MATOS et al., 2010), e promover atendimento que vise ao bem estar e a qualidade de vida daquelas que já têm o diagnóstico (BRASIL, 2010; 2014). Sendo assim, o objetivo do presente estudo foi de investigar as associações entre nível socioeconômico na incidência por câncer de mama.

\section{MATERIAL E MÉTODO}

Trata-se de revisão sistemática de literatura científica, realizada conforme as recomendações metodológicas da declaração PRISMA - Preferred Reporting Items for Systematic Reviews and MetaAnalyses (MOHER et al., 2009; LIBERATI et al., 2009).

Os descritores utilizados na busca realizada entre fevereiro e março de 2016 foram: nível socioeconômico, neoplasias da mama, classe social, mortalidade, nas bases de dados da Biblioteca Virtual em Saúde (BVS), Scielo, Medline, Biblioteca Cochrane, LILACS e US National Library of Medicine National Institutes of Health (PUBMED).

Os critérios de inclusão foram: publicações de 2010 a 2015; em periódicos nacionais e internacionais; publicados em português, inglês e espanhol e aqueles encontrados na íntegra. Os estudos que não obedeceram aos critérios de inclusão supracitados foram excluídos da pesquisa. Estudos referentes a abordagem prioritária de fatores genéticos, abordagem de tipos específicos de neoplasias, outros tipos de neoplasia, artigos de opiniões e comentários, dissertações, amostra não 
significativa e informações repetidas foram utilizados como critérios de exclusão.

Os artigos científicos coletados foram analisados considerando as seguintes categorias: 1) ano, 2) base de dados; 3) tipo de pesquisa; 4) fatores socioeconômicos; 5) incidência por câncer de mama; 6) mortalidade por esta doença; e 7) idioma de publicação.

\section{RESULTADO}

Inicialmente, identificaram-se 7.300 itens. Ao se refinar a busca a partir dos descritores e critérios de inclusão, o número foi redefinido para 271. Desses, após leitura do título e resumo, 196 apresentaram tema diferente do objetivado e 13 eram dissertações. Assim, 62 artigos foram lidos na íntegra, dos quais 32 foram excluídos conforme os critérios estabelecidos. Foram selecionados 30 artigos para análise da revisão sistemática, conforme Figura 1.

FIGURA 1 - Descrição dos resultados de acordo com método PRISMA.

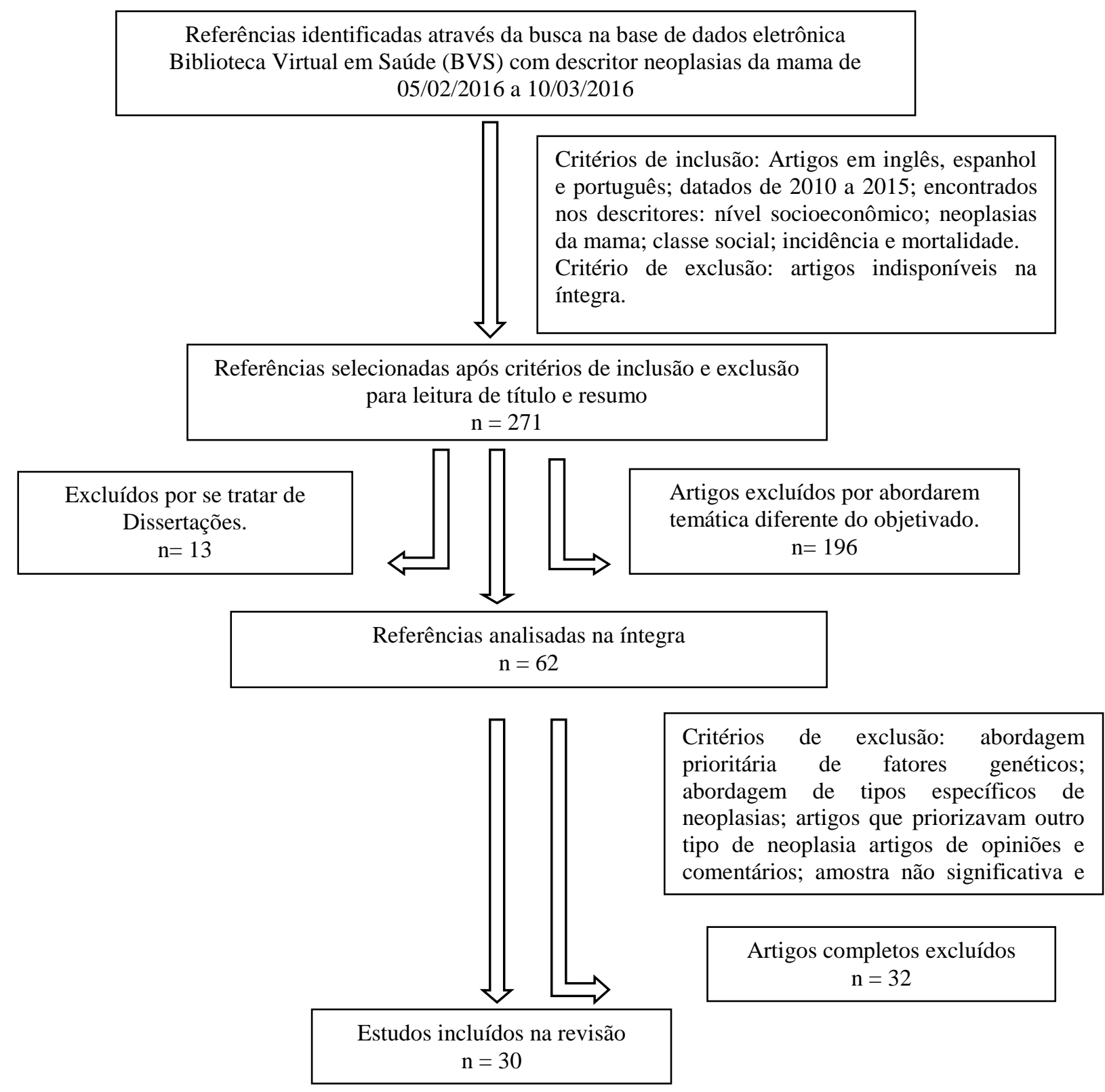

Fonte: Elaborado pelos autores 
As características dos artigos analisados nesse estudo foram sumarizadas na Tabela 1.

Tabela 1 - Características dos artigos selecionados.

(Continua)

\begin{tabular}{|c|c|c|c|c|}
\hline Título & Ano & Nac. & Periódico & Método \\
\hline $\begin{array}{l}\text { Mortalidade por câncer de mama em hospital de } \\
\text { referência em oncologia }\end{array}$ & 2013 & Brasil & $\begin{array}{l}\text { Rev Bras } \\
\text { Epidemiol }\end{array}$ & Transversal \\
\hline $\begin{array}{l}\text { Educating Low SES and LEP Survivors About } \\
\text { Breast Cancer Research }\end{array}$ & 2015 & EUA & J Cancer educ & $\begin{array}{l}\text { Participativa } \\
\text { qualitativa }\end{array}$ \\
\hline $\begin{array}{l}\text { Disparities in race/ethnicity and socioeconomic } \\
\text { status }\end{array}$ & 2013 & EUA & BMC Cancer. & Transversal \\
\hline $\begin{array}{l}\text { Desigualdades associadas à não realização de } \\
\text { mamografia na }\end{array}$ & 2012 & Brasil & $\begin{array}{l}\text { Rev Bras } \\
\text { Epidemiol }\end{array}$ & Transversal \\
\hline \multicolumn{5}{|l|}{ zona urbana de Teresina-Piauí- Brasil } \\
\hline $\begin{array}{l}\text { Effect of socioeconomic status as measured by } \\
\text { education level on survival in breast cancer } \\
\text { clinical trials }\end{array}$ & 2011 & EUA & $\begin{array}{l}\text { Psychooncolo } \\
\text { gy }\end{array}$ & Transversal \\
\hline $\begin{array}{l}\text { Breast cancer incidence and case fatality among } \\
4.7 \text { million women in relation to social and ethnic } \\
\text { background }\end{array}$ & 2012 & EUA & $\begin{array}{l}\text { Breast Cancer } \\
\text { Res. }\end{array}$ & Coorte \\
\hline $\begin{array}{l}\text { Individual and Neighborhood Socioeconomic } \\
\text { Status in Relation to Breast Cancer Incidence in } \\
\text { African American Women }\end{array}$ & 2012 & EUA & $\begin{array}{l}\text { Am J } \\
\text { Epidemiol. }\end{array}$ & Coorte \\
\hline $\begin{array}{l}\text { Small but significant socioeconomic inequalities } \\
\text { in axillary staging and treatment of breast cancer } \\
\text { in the Netherlands }\end{array}$ & 2012 & $\begin{array}{l}\text { Reino } \\
\text { Unido }\end{array}$ & Br J Cancer. & Transversal \\
\hline $\begin{array}{l}\text { Inflammatory and non-inflammatory breast cancer } \\
\text { survival by socioeconomic position in the } \\
\text { Surveillance, Epidemiology, and End Results } \\
\text { database, 1990-2008 }\end{array}$ & 2012 & EUA & $\begin{array}{l}\text { Breast Cancer } \\
\text { Res Treat }\end{array}$ & Transversal \\
\hline $\begin{array}{l}\text { Breast cancer stage at diagnosis and area based } \\
\text { socioeconomic status: a }\end{array}$ & 2012 & China & BMC Cancer. & $\begin{array}{l}\text { Transversal } \\
\text { retrospectivo }\end{array}$ \\
\hline \multicolumn{5}{|l|}{$\begin{array}{l}\text { multicenter 10year retrospective clinical } \\
\text { epidemiological study in China }\end{array}$} \\
\hline $\begin{array}{l}\text { Healthcare access and mammography screening in } \\
\text { Michigan }\end{array}$ & 2012 & EUA & $\begin{array}{l}\text { Int J Equity } \\
\text { Health }\end{array}$ & Transversal \\
\hline $\begin{array}{l}\text { Socioeconomic disparities in the uptake of breast } \\
\text { and cervical cancer screening in Italy }\end{array}$ & 2012 & Itália & $\begin{array}{l}\text { BMC Public } \\
\text { Health. }\end{array}$ & Transversal \\
\hline $\begin{array}{l}\text { Fatores associados à realização da prevenção } \\
\text { secundária do câncer de mama no Município de } \\
\text { Maringá, Paraná, Brasil }\end{array}$ & 2011 & Brasil & $\begin{array}{l}\text { Cad. Saúde } \\
\text { Pública }\end{array}$ & Transversal \\
\hline $\begin{array}{l}\text { Decline in US Breast Cancer Rates After the } \\
\text { Women's Health Initiative }\end{array}$ & 2010 & EUA & $\begin{array}{l}\text { Am J Public } \\
\text { Health. }\end{array}$ & Transversal \\
\hline $\begin{array}{l}\text { The Modifying Effect of Social Class on the } \\
\text { Relationship Between Body Mass Index and } \\
\text { Breast Cancer Incidence }\end{array}$ & 2010 & EUA & $\begin{array}{l}\text { Am J Public } \\
\text { Health. }\end{array}$ & Coorte \\
\hline $\begin{array}{l}\text { Are caregiving responsibilities associated with } \\
\text { nonattendance at breast screening? }\end{array}$ & 2010 & Irlanda & $\begin{array}{l}\text { BMC Public } \\
\text { Health. }\end{array}$ & Coorte \\
\hline $\begin{array}{l}\text { Mortalidade por câncer de mama e câncer de colo } \\
\text { do útero em município de porte médio da Região } \\
\text { Sudeste do Brasil, } 1980 \text { - } 2006\end{array}$ & 2011 & Brasil & $\begin{array}{l}\text { Cad. Saúde } \\
\text { Pública }\end{array}$ & Transversal \\
\hline
\end{tabular}


Tabela 1 - Características dos artigos selecionados.

(Conclusão)

\begin{tabular}{|c|c|c|c|c|}
\hline Título & Ano & Nac. & Periódico & Método \\
\hline $\begin{array}{l}\text { Breast cancer and early retirement: associations } \\
\text { with disease characteristics, treatment, } \\
\text { comorbidity, social position and participation in a } \\
\text { six day rehabilitation course in a register based } \\
\text { study in Denmark }\end{array}$ & 2011 & $\begin{array}{l}\text { Dina- } \\
\text { marca }\end{array}$ & $\begin{array}{l}\text { Acta } \\
\text { Oncologica }\end{array}$ & $\begin{array}{l}\text { Transversal } \\
\text { retrospecticvo }\end{array}$ \\
\hline $\begin{array}{l}\text { Changes over time in socioeconomic inequalities } \\
\text { in breast and rectal cancer survival in England and } \\
\text { Wales during a 32year period (1973-2004) }\end{array}$ & 2011 & $\begin{array}{l}\text { Reino } \\
\text { Unido }\end{array}$ & $\begin{array}{l}\text { Annals of } \\
\text { Oncology }\end{array}$ & Transversal \\
\hline $\begin{array}{l}\text { Estudo da acessibilidade ao serviço de saúde de } \\
\text { pacientes com câncer de mama no município de } \\
\text { campos dos Goytacazes. }\end{array}$ & 2011 & Brasil & $\begin{array}{l}\text { Cad. Saúde } \\
\text { Pública }\end{array}$ & Retrospectivo \\
\hline $\begin{array}{l}\text { Impact of neighborhood and individual } \\
\text { socioeconomic status on survival after } \\
\text { breast cancer varies by race/ethnicity }\end{array}$ & 2014 & EUA & $\begin{array}{l}\text { Cancer } \\
\text { Epidemiol } \\
\text { Biomarkers } \\
\text { Prev. }\end{array}$ & Transversal \\
\hline $\begin{array}{l}\text { Impact of neighborhood racial composition and } \\
\text { metropolitan residential segregation on disparities } \\
\text { in breast cancer stage at diagnosis and survival } \\
\text { between black and white women in California }\end{array}$ & 2010 & EUA & $\begin{array}{l}\text { J Community } \\
\text { Health. }\end{array}$ & Transversal \\
\hline $\begin{array}{l}\text { Income and Long-Term Breast Cancer Survival: } \\
\text { Comparisons of Vulnerable Urban Places in } \\
\text { Ontario and California }\end{array}$ & 2010 & $\begin{array}{l}\text { Cana- } \\
\text { dá }\end{array}$ & Breast J. & Coorte \\
\hline $\begin{array}{l}\text { Influence of Race, Insurance, Socioeconomic } \\
\text { Status, and Hospital Type on Receipt of Guideline } \\
\text { Concordant Adjuvant Systemic Therapy for } \\
\text { Locoregional Breast Cancers }\end{array}$ & 2010 & EUA & $\begin{array}{l}\text { Journal of } \\
\text { Clinical } \\
\text { Oncology }\end{array}$ & Transversal \\
\hline $\begin{array}{l}\text { How much of the deprivation gap in cancer } \\
\text { survival can be explained by variation in stage at } \\
\text { diagnosis }\end{array}$ & 2013 & $\begin{array}{l}\text { Reino } \\
\text { Unido }\end{array}$ & $\begin{array}{l}\text { International } \\
\text { Journal of } \\
\text { Cancer }\end{array}$ & Transversal \\
\hline $\begin{array}{l}\text { Socioeconomic inequalities in breast and cervical } \\
\text { cancer screening practices in Europe }\end{array}$ & 2010 & $\begin{array}{l}\text { Espa- } \\
\text { nha }\end{array}$ & $\begin{array}{l}\text { International } \\
\text { Journal of } \\
\text { Epidemiology }\end{array}$ & Transversal \\
\hline $\begin{array}{l}\text { Desigualdades socioeconômicas na incidência e } \\
\text { mortalidade por câncer: revisão de estudos } \\
\text { ecológicos, 1998-2008 }\end{array}$ & 2013 & Brasil & $\begin{array}{l}\text { Saúde e } \\
\text { Sociedade }\end{array}$ & $\begin{array}{l}\text { Revisão } \\
\text { sistemática }\end{array}$ \\
\hline $\begin{array}{l}\text { Socioeconomic status and survival after an } \\
\text { invasive breast cancer diagnosis }\end{array}$ & 2012 & EUA & Câncer. & Caso controle \\
\hline $\begin{array}{l}\text { Acesso à assistência oncológica: mapeamento dos } \\
\text { fluxos origem-destino das internações e dos } \\
\text { atendimentos ambulatoriais. O caso do câncer de } \\
\text { mama }\end{array}$ & 2011 & Brasil & $\begin{array}{l}\text { Cad. Saúde } \\
\text { Pública }\end{array}$ & $\begin{array}{l}\text { Estudo } \\
\text { ecológico }\end{array}$ \\
\hline $\begin{array}{l}\text { The Association of Socioeconomic Status and } \\
\text { Access to Low Volume Service Providers in } \\
\text { Breast Cancer }\end{array}$ & 2013 & China & PLoS One. & Transversal \\
\hline
\end{tabular}

Fonte: Elaborado pelos autores.

A maior concentração de publicações ocorreu em 2012 (30\%), seguido de 2011 (23,3\%) e 2010 (23,3\%), 2013 (16,7\%), 2014 (3,1\%) e 2015 (3,1\%). 
Estados Unidos e Brasil apresentaram 40,6\% e $28,1 \%$, respectivamente, do total das publicações; Reino Unido, 9,4\%, China, 6,2\%, Dinamarca, Espanha, Itália, Irlanda e Canadá, cada um, 3,3\%. Identificou-se a língua inglesa em 76,7\% dos artigos e a portuguesa, em 23,3\%.

Quanto ao método dos estudos predominaram os transversais $(60 \%)$ seguidos pelos de coorte $(16,7 \%)$, transversal retrospectivo $(6,7 \%)$. Estudos de caso controle, ecológico, retrospectivo, revisão sistemática de literatura e pesquisa participativa qualitativa colaboraram com $3,3 \%$, cada um deles.

\section{DISCUSSÃO}

Albrecht et al. constataram que entre os fatores relacionados à prevenção primária do câncer de mama, o baixo grau de instrução esteve fortemente associado ao estadiamento mais avançado e maiores chances de metástases, pois mulheres com menor escolaridade estão mais propensas a um diagnóstico tardio quando comparadas a mulheres de classes mais altas e com maior nível de instrução. Schlichting et al. (2012) apontaram que menor renda, bem como a baixa escolaridade estão associadas ao diagnóstico mais tardio. Comparando o grupo de mulheres que possui seguro de saúde e o grupo que não possui, as primeiras são mais propensas a receber cirurgia conservadora, enquanto aquelas pertencentes ao segundo grupo são submetidas à mastectomia da mama, justamente por serem diagnosticadas em um estágio mais avançado da doença (SCHLICHTING et al., 2012). Rodrigues et al. (2011) relatam maior sobrevida nas pacientes pertencentes a uma classe econômica mais alta, pois mulheres de classes mais baixas possuem limitações no acesso a serviços de saúde dificultando o diagnóstico precoce e, portanto, impossibilitando a oportunidade de receberem tratamento adequado a tempo de se obter a cura. Aarts et al. (2012) observaram que mulheres com um nível socioeconômico mais baixo apresentaram maiores taxas de dissecção de linfonodos, ou seja, o câncer foi diagnosticado em um estágio mais avançado.

De acordo com Barros et al., o atraso no diagnóstico do câncer de mama tem importante impacto na sobrevida, principalmente quando o tratamento específico atrasa mais de três meses após a primeira identificação da sintomalogia. Segundo Wang et al. (2012), a fase em que o câncer de mama foi diagnosticado é um indicador de acesso aos cuidados de saúde. Mulheres residentes de áreas com um Produto Interno Bruto menor e com alta porcentagem de analfabetismo são diagnosticadas mais tardiamente, e após o diagnóstico tem maior dificuldade no acesso aos cuidados de saúde, como tratamento e acompanhamento. Sprague et al. (2011) mostraram forte associação entre nível educacional e mortalidade, pois mulheres que vivem em áreas nas quais pelo $20 \%$ dos adultos não completaram o ensino médio tem aproximadamente 1,6 vezes mais chance de morrer pelo câncer de mama do que mulheres que vivem em áreas em que menos de $10 \%$ dos adultos não possuem um diploma do ensino médio. Segundo Herndon et al. (2013), pacientes que não completaram o ensino médio possuem pior prognóstico do que aquelas que concluíram o ensino médio.

Sharriff-Marco et al. (2014) evidenciaram que o nível socioeconômico se relaciona ao pior prognóstico independente do nível de escolaridade e de outros fatores, como a etnia, por exemplo. Essa relação entre nível socioeconômico e mortalidade pelo câncer de mama também foi evidenciada por Matos et al. (2010) em que a classe econômica e a escolaridade se ressaltaram como os fatores que mais interferiram nas condutas preventivas, tanto na mamografia como na realização do exame clínico. Mulheres de classes mais baixas mostraram-se menos propensas a realizar dos exames preventivos (MATOS, 2010). Mulheres de baixa renda, conforme $\mathrm{Wu}$ et al., não costumam procurar cuidados proativamente, pela falta de informação e pela dificuldade em se comunicar. Segundo Chang et al. (2013), além da falta de recursos, pacientes de nível socioeconômico mais baixo estão sujeitas a desenvolver depressão e se isolar socialmente devido a falta de informações acerca da doença. 
A comparar mulheres diagnosticadas com neoplasia mamária em dois bairros urbanos, um localizado no Canadá e outro nos Estados Unidos, ambos com uma população de nível socioeconômico mais baixo, Gorey et al. (2010) observaram que o bairro canadense apresentou 15 anos de sobrevivência a mais em relação ao bairro americano. Concluíram que a diferença se dá pela maior facilidade de acesso aos serviços de saúde por parte dos canadenses. Palencia et al. (2010) observaram que quando o programa de rastreio é eficaz e há facilidade no acesso aos serviços de saúde, as desigualdades sociais não alteram de maneira significativa os padrões de mortalidade. Essa relação entre acessibilidade aos serviços de saúde e prognóstico e mortalidade da doença também foi exposta por Rodrigues e Bustamante-Teixeira (2011) em que apenas $10 \%$ das mulheres diagnosticadas em estágio IV da doença conseguiram atendimento especializado em um período de doze meses. De acordo com Oliveira et al. (2011), a escassez de recursos e a dificuldade de acesso aos métodos diagnósticos e terapêuticos estão fortemente associados à mortalidade e essa carência ocorre mesmo nas regiões brasileiras em que a oferta de serviços é maior. No entanto, ela fica mais evidente em regiões menos povoadas e com maiores dificuldades de acesso aos serviços de saúde, como a região Norte, onde aproximadamente metade dos municípios não encaminhou qualquer paciente para a cirurgia de mama na rede pública e conveniada ao SUS; 17,8\% não encaminharam para a quimioterapia; e $49 \%$ não conduziram pacientes para a radioterapia (OLIVEIRA, et al., 2011).

Warner e Gomes (2010) analisaram a sobrevida de mulheres brancas e negras residentes em bairros ricos e mulheres brancas e negras residentes de bairros mais pobres com câncer de mama. O status socioeconômico foi mais associado à sobrevida do que a raça, ou seja, moradoras de bairros mais pobres, negras ou brancas, tiveram diagnóstico mais tardio e consequentemente possuíram uma pior sobrevida do que aquelas que residiam em bairros mais ricos. Akinyemiju et al. (2012) observaram que as taxas de triagem são mais baixas em mulheres negras do que em mulheres brancas, e concluíram, assim como Warner et al., que a raça não interfere diretamente na sobrevida da doença, e sim os recursos e o acesso aos serviços de saúde.

Parise e Caggiano (2013) não constataram diferença significativa no ano de diagnóstico independente do status socioeconômico. Nos estágios iniciais do câncer também não houve disparidades na mortalidade nas diferentes camadas socioeconômicas, no entanto, mulheres com nível socioeconômico mais elevado em estágios mais avançados da doença apresentaram maior mortalidade. Rutherford et al. (2013) observaram que 40 mortes no leste da Inglaterra e 450 mortes em todo o país, seriam adiadas para um tempo posterior a cinco anos se as privações devido aos status socioeconômico fossem eliminadas.

Dentre todos os fatores estudados na realização da prevenção secundária do câncer de mama, a classe econômica é o único fator em comum que interfere nas condutas preventivas: mulheres que pertencem às camadas mais baixas realizam a mamografia com menor frequência e são menos submetidas ao exame clínico das mamas; mulheres com nove anos de estudos ou mais eram examinadas com mais periodicidade do que aquelas que estudaram por até quatro anos (PEREIRA et al., 2014). Damkjaer et al. (2011) apontaram que um nível socioeconômico mais baixo implica em um aumento do risco para o câncer de mama.

Kinnear et al. (2010) verificaram forte relação entre a classe econômica e a participação nos programas de triagem. As classes econômicas mais baixas estiveram 66\% menos propensas a participar dos programas de prevenção do que as mais altas. Damiani et al. (2012) analisaram a associação do nível de escolaridade e os programas de rastreio do câncer de mama. Baixo nível de escolaridade esteve associado a uma subutilização rastreamento, ou seja, a adesão aos programas de rastreio foi maior entre as mais escolarizadas. No entanto, uma vez participantes dos programas, as menos escolarizadas participaram de maneira mais organizada e periódica. 
Beiki et al. (2012) observaram que mulheres com maior nível de escolaridade tinham significativamente maior incidência de câncer de mama, de $20 \%$ a $30 \%$ mais elevada, em comparação com aquelas com menor escolaridade, o que pode estar associado aos casos subdiagnosticados entre aquelas com menor escolaridade. Conforme Lages et al. (2012), uma mulher analfabeta apresenta quase sete vezes chances de não realizar a mamografia.

Ribeiro et al. (2013), Torio et al. (2010) e Palmer (2012), verificaram associação entre a incidência de câncer de mama e um nível socioeconômico elevado, devido a uma maior prevalência de fatores de risco reprodutivos nas classes mais altas, como menor idade da menarca, menor paridade, idade maior na primeira gestação, entre outros. Atribui-se essa relação à presença dos fatores de risco reprodutivos, já que essa incidência foi maior apenas nos tumores estrógenos positivos. Já Krieger et al. (2010) verificaram uma queda na incidência da doença nas mulheres de renda mais alta. No entanto, observaram que essa queda coincidiu com uma redução no uso de terapia hormonal, não se sabendo, portanto, se essa queda teve relação direta com a classe econômica ou com a diminuição do uso de terapia hormonal, também considerado fator de risco, e as classes mais altas são as principais usuárias de terapias hormonais.

\section{CONCLUSÃO}

O nível socioeconômico exerce influência nas práticas de prevenção secundária do câncer de mama, de modo que grande parte dos trabalhos apontou que quanto mais alto o nível socioeconômico da mulher, maior a prevalência de realização das condutas preventivas. Observou-se também relação entre escolaridade e mortalidade pelo câncer de mama. Mulheres com maior nível de escolaridade tendem a ser mais ativas na procura por informação e por atendimento, possuindo maior facilidade em entender e em se fazer entender, propiciando maiores chances de diagnóstico precoce.

Estudos sobre as dificuldades do acesso aos serviços de saúde, bem como das consequências do atraso no diagnóstico e no tratamento do câncer de mama ainda são escassos no Brasil. Pesquisas internacionais apresentam um cenário de saúde diferente do Brasil, dificultando a aplicação dos resultados para a realidade nacional.

\section{AGRADECIMENTOS}

À universidade pela concessão da bolsa de Iniciação Científica e ao Instituto de Pesquisa pelos incentivos aos pesquisadores.

\section{REFERÊNCIAS}

AARTS MJ.et al. Small but significant socioeconomic inequalities in axillary staging and treatment of breast cancer in the Netherlands. British journal of cancer, v.7, n.1, p.12-17, 2012.Disponível em: https://www.ncbi.nlm.nih.gov/pubmed/22596236. Acesso em 16 jul. 2019.

AKINYEMIJU, T. F.et al. Healthcare access and mammography screening in Michigan: a multilevel cross-sectional study. Int J Equity Health. 112012. p. 16. 2012

https://www.ncbi.nlm.nih.gov/pmc/articles/PMC3414751/ Acesso em 16 jul. 2019. 
ALBRECHT, C. A.M.et al. Breast cancer mortality among patients attending a cancer hospital, Vitoria, ES. Rev. bras. epidemiol., São Paulo, v. 16, n. 3, p. 582-591, Set. 2013.Disponível em: http://www.scielo.br/scielo.php?script=sci_arttext\&pid=S1415-790X2013000300582\&lng=en\&nrm=iso. Acesso em 16 jul. 2019.

BARROS , Â. F.; UEMURA G.; MACEDO, J. L. Interval for access to treatment for breast cancer in the Federal District, Brazil. Rev Bras Ginecol Obstet., v.35, n.10, p.458-463. Disponível em: http://www.scielo.br/scielo.php?script=sci_arttext\&pid=S0100-72032013001000006\&lng=en\&nrm=iso. Acesso em 16 jul. 2019.

BARRY, J.; BREEN, N. The importance of place of residence in predicting late-stage diagnosis of breast or cervical cancer. Health \& place, v.11, n.1, p.15-29, 2005. Disponível em: https://www.ncbi.nlm.nih.gov/pubmed/15550353. Acesso em 19 jul. 2019.

BEIKI, O.; HALL, P.; EKBOM, A.; MORADI, T. Breast cancer incidence and case fatality among 4.7 million women in relation to social and ethnic background: a population-based cohort study. Breast cancer research: BCR, 14(1):R5, 2012. Disponível em: https://www.ncbi.nlm.nih.gov/pubmed/22225950. Acesso em 19 jul. 2019.

BRASIL. Ministério da Saúde. Instituto Nacional de Câncer. Câncer Tipo Mama. 2019. Disponível em https://www.inca.gov.br/tipos-de-cancer/cancer-de-mama. Acesso em: em 16 jul. 2019.

BRASIL. Ministério da Saúde. Política Nacional de Promoção da Saúde. 3 ed. Brasília, 2010.

BRASIL. Ministério da Saúde. Portaria No 2.446, de 11 de Novembro de 2014. 2014. Disponível em: http://bvsms.saude.gov.br/bvs/saudelegis/gm/2014/prt2446_11_11_2014.html. Acesso em 19 jul. 2019.

CHANG, C. M.et al. The Association of Socioeconomic Status and Access to Low-Volume Service Providers in Breast Cancer. PLoS One. e81801, 2013. Disponível em: https://journals.plos.org/plosone/article?id=10.1371/journal.pone.0081801. Acesso em 16 jul. 2019.

CINTRA, J. R. D.; GUERRA, M.R.; BUSTAMANTE-TEIXEIRA, M. T. Non-metastatic breast cancer specific-survival of patients after treatment with adjuvant chemotherapy. Rev. Assoc. Med. Bras., v. 54, n. 4, p. 339-346, 2008. Disponível em http://www.scielo.br/scielo.php?script=sci_arttext\&pid=S0104-42302008000400020\&lng=en\&nrm=iso. Acesso em: 16 jul. 2019.

DAMIANI, G.et al. Socioeconomic disparities in the uptake of breast and cervical cancer screening in Italy: a cross sectional study. BMC public health,12:99, 2012, Disponível em: https://bmcpublichealth.biomedcentral.com/articles/10.1186/1471-2458-12-99. Acesso em: 19 jul. 2019.

DAMKJAER, L. H.et al. Breast cancer and early retirement: Associations with disease characteristics, treatment, comorbidity, social position and participation in a six-day rehabilitation course in a registerbased study in Denmark. Acta oncologica (Stockholm, Sweden), v.50, n.2, p.274-281, 2011. https://www.ncbi.nlm.nih.gov/pubmed/21231788. Acesso em 19 jul. 2019.

FACINA, T. Estimativa 2014. Incidência de Câncer no Brasil Estimate 2014. Rev bras cancerol, v.60, n.1, p.63-64, 2014. http://www1.inca.gov.br/rbc/n_60/v01/pdf/11-resenha-estimativa-2014-incidencia-decancer-no-brasil.pdf. Acesso em 16 jul. 2019

FELIX, J.D.et al. Avaliação da completude das variáveis epidemiológicas do Sistema de Informação sobre Mortalidade em mulheres com óbitos por câncer de mama na Região Sudeste: Brasil (1998 a 2007 ). Ciência \& Saúde Coletiva, Rio de Janeiro, v.17, n.4, p.945-953, 2012. 
GONÇALVES, A. T. C.et al. Increase in breast cancer mortality in Southern Brazil from 1980 to 2002. Cad. Saúde Pública, v. 23, n. 8, p. 1785-1790, 2007. Disponível em http://www.scielo.br/scielo.php?script=sci_arttext\&pid=S0102-311X2007000800005\&lng=en\&nrm=iso. Acesso em 16 jul. 2019

GOREY KM.et al. Income and Long-Term Breast Cancer Survival: Comparisons of Vulnerable Urban Places in Ontario and California. Breast J. v.16, n.4, p.416-419, 2010. Disponível em: https://www.ncbi.nlm.nih.gov/pmc/articles/PMC2919557/. Acesso em 16 jul. 2019.

HERNDON, J. E.et al.Effect of socioeconomic status as measured by education level on survival in breast cancer clinical trials. Psycho-oncology, v.22, n.2, p.315-323, 2013. Disponível em: https://www.ncbi.nlm.nih.gov/pubmed/22021121. Acesso em 16 jul. 2019.

HÖFELMANN, D. A.et al. Sobrevida em dez anos e fatores prognósticos em mulheres com câncer de mama em Joinville, Santa Catarina, Brasil. Ciênc. saúde coletiva, Rio de Janeiro , v. 19, n. 6, p. 18131824, 2014 . Disponível em: http://www.scielo.br/scielo.php?script=sci_arttext\&pid=S141381232014000601813\&lng=en\&nrm=iso. Acesso em: 16 jul. 2019.

KINNEAR, H.et al. Are caregiving responsibilities associated with non-attendance at breast screening? BMC public health, 10:749, 2010. Disponível em: https://www.ncbi.nlm.nih.gov/pubmed/21129196. Acesso em 19 jul. 2019.

KRIEGER, N.; CHEN, J. T.; WATERMAN, P. D. Decline in US breast cancer rates after the Women's Health Initiative: socioeconomic and racial/ethnic differentials. American journal of public health. 100 Suppl 1:S132-9, 2010. Disponível em: https://www.ncbi.nlm.nih.gov/pmc/articles/PMC2837433/. Acesso em 19 jul. 2019.

LAGES, R. B.et al.Inequalities associated with lack of mammography in Teresina-Piauí-Brazil, 20102011. Rev bras epidemiol, v. 15, n. 4, p. 737-747, 2012. Disponível em: http://www.scielo.br/scielo.php?script=sci_arttext\&pid=S1415-790X2012000400006\&lng=en\&nrm=iso. Acesso em: 16 July 2019.

LIBERATI, A. et al. The PRISMA statement for reporting systematic reviews and meta-analyses of studies that evaluate healthcare interventions: explanation and elaboration. 2009. BMJ, 339 :b2700, 2009. Disponível em: https://www.bmj.com/content/339/bmj.b2700. Aceso em; 16 jul. 2019.

MATOS, J. C.et al. Mortalidade por câncer de mama em mulheres do município de Maringá, Paraná, Brasil. Revista Gaúcha de Enfermagem, v.30, m.3, p.445-452, 2010. Disponível em: https://seer.ufrgs.br/RevistaGauchadeEnfermagem/article/view/8989/6994. Acesso em 19 jul. 2019.

MOHER, D.et al.. Preferred reporting items for systematic reviews and meta-analyses: the PRISMA statement. Annals of internal medicine. v.151, n.4, p. 264-269, 2009.

OLIVEIRA, E. X.G.et al. Access to cancer care: mapping hospital admissions and high-complexity outpatient care flows. The case of breast cancer. Cad. Saúde Pública, Rio de Janeiro, v. 27, n. 2, p. 317 326, 2011. Disponível em: http://www.scielo.br/scielo.php?script=sci arttext\&pid=S0102311X2011000200013\&lng=en\&nrm=iso. Acesso em 16 jul. 2019

PALENCIA, L.et al. Socio-economic inequalities in breast and cervical cancer screening practices in Europe: influence of the type of screening program. International journal of epidemiology, v.39, n.3, p.757-765, 2010. Disponível em: https://www.ncbi.nlm.nih.gov/pubmed/20176587. Acesso em 16 jul. 2019.

PALMER, J. R.et al. Individual and neighborhood socioeconomic status in relation to breast cancer incidence in African-American women. American journal of epidemiology. V.176, n.12, p.1141-1146, 2012. Disponível em: https://www.ncbi.nlm.nih.gov/pubmed/23171873. Acesso em 19 jul. 2019. 
PARISE, C. A.; CAGGIANO, V. Disparities in race/ethnicity and socioeconomic status: risk of mortality of breast cancer patients in the California Cancer Registry, 2000-2010. BMC cancer. p.13:449. 2013. Disponível em: https://www.ncbi.nlm.nih.gov/pubmed/24083624. Acesso em 19 jul. 2019.

PEREIRA, M. B.et al. Recommended age groups and frequency of mammography screening: a systematic review. Ciênc. saúde coletiva, v.19, n.4, p.1135-1140, 2014. Disponível em: https://www.ncbi.nlm.nih.gov/pubmed/24820596. Acesso em 19 jul. 2019.

RENCK, D. V.et al. Equidade no acesso ao rastreamento mamográfico do câncer de mama com intervenção de mamógrafo móvel no sul do Rio Grande do Sul, Brasil.Cad. Saúde Pública, Rio de Janeiro , v. 30, n. 1, p. 88-96, 2014 . Disponível em: http://www.scielo.br/scielo.php?script=sci_arttext\&pid=S0102-311X2014000100088\&lng=en\&nrm=iso. Acesso em 19 jul. 2019.

RIBEIRO, A. A.; NARDOCCI, A.C. Socioeconomic inequalities in cancer incidence and mortality: review of ecological studies, 1998-2008. Saude soc. v. 22, n. 3, p. 878-891, 2013. Disponível em: http://www.scielo.br/scielo.php?script=sci_arttext\&pid=S0104-12902013000300020\&lng=en\&nrm=iso. Acesso em:16 jul. 2019.

RODRIGUES, A. D.; BUSTAMANTE-TEIXEIRA, M. T. Breast cancer and cervical cancer mortality trends in a medium-sized city in Southern Brazil, 1980-2006. Cad Saúde Pública, v.27, n.2, p.241-248, 2011. Disponível em: http://www.scielo.br/scielo.php?script=sci abstract\&pid=S0102311X2011000200005\&lng=en\&nrm=iso\&tlng=en. Acesso em 16 jul. 2019.

RUTHERFORD, M. J.et al. How much of the deprivation gap in cancer survival can be explained by variation in stage at diagnosis: an example from breast cancer in the East of England. International journal of cancer, v.133, n.9, p.2192-2200, 2013. Disponível em:

https://www.ncbi.nlm.nih.gov/pubmed/23595777. Acesso em 19 jul. 2019.

SCHLICHTING, J. A.et al. Inflammatory and non-inflammatory breast cancer survival by socioeconomic position in the Surveillance, Epidemiology, and End Results database, 1990-2008. Breast cancer research and treatment, v.134, n.3, p.1257-1268, 2012. Disponível em: https://www.ncbi.nlm.nih.gov/pmc/articles/PMC4291081/. Acesso em 16 jul. 2019.

SHARIFF-MARCO, S et al. Impact of neighborhood and individual socioeconomic status on survival after breast cancer varies by race/ethnicity: the Neighborhood and Breast Cancer Study. Cancer epidemiology, biomarkers \& prevention : a publication of the American Association for Cancer Research, cosponsored by the American Society of Preventive Oncology, v.23, n.5, p.793-811, 2014. Disponível em: https://www.ncbi.nlm.nih.gov/pubmed/24618999. Acesso em 16 jul. 2019.

SILVA, G. A.et al. Access to early breast cancer diagnosis in the Brazilian Unified National Health System: an analysis of data from the Health Information System. Cad Saúde Pública, Rio de Janeiro, v. 30, n. 7, p. 1537-1550, Jul. 2014 . Disponível em:

http://www.scielo.br/scielo.php?script=sci_arttext\&pid=S0102-311X2014000701537\&lng=en\&nrm=is. Acesso em: 16 jul. 2019.

SPRAGUE, B. L. et al. Socioeconomic status and survival after an invasive breast cancer diagnosis.

Cancer. v.117, n7, p.1542-1551, 2011. Disponível em: https://www.ncbi.nlm.nih.gov/pubmed/21425155. Acesso em 16 jul. 2019.

TORIO, C. M.et al. The modifying effect of social class on the relationship between body mass index and breast cancer incidence. American journal of public health. v.100, n.1, p.146-151, 2010. Disponível em: https://www.ncbi.nlm.nih.gov/pmc/articles/PMC2791249/. Acesso em: 16 jul. 2019

WANG, Q.; LI, J.et al. Breast cancer stage at diagnosis and area-based socioeconomic status: a multicenter 10-year retrospective clinical epidemiological study in China. BMC cancer. v.12, n.1, p.122, 2012. Disponível em: https://www.ncbi.nlm.nih.gov/pubmed/22455370. Acesso em 16 jul. 2019. 
WARNER, E. T.; GOMEZ, S. L. Impact of neighborhood racial composition and metropolitan residential segregation on disparities in breast cancer stage at diagnosis and survival between black and white women in California. Journal of community health. v.35, n.4, p.398-408, 2010. Disponível em:

https://www.ncbi.nlm.nih.gov/pubmed/20358266. Acesso em 16 jul. 2019.

WU, X. C.et al. Influence of race, insurance, socioeconomic status, and hospital type on receipt of guideline-concordant adjuvant systemic therapy for locoregional breast cancers. Journal of clinical oncology : official journal of the American Society of Clinical Oncology. 2012;30(2):142-50. Disponível em: https://www.ncbi.nlm.nih.gov/pubmed/22147735. Acesso em 16 jul. 2019. 